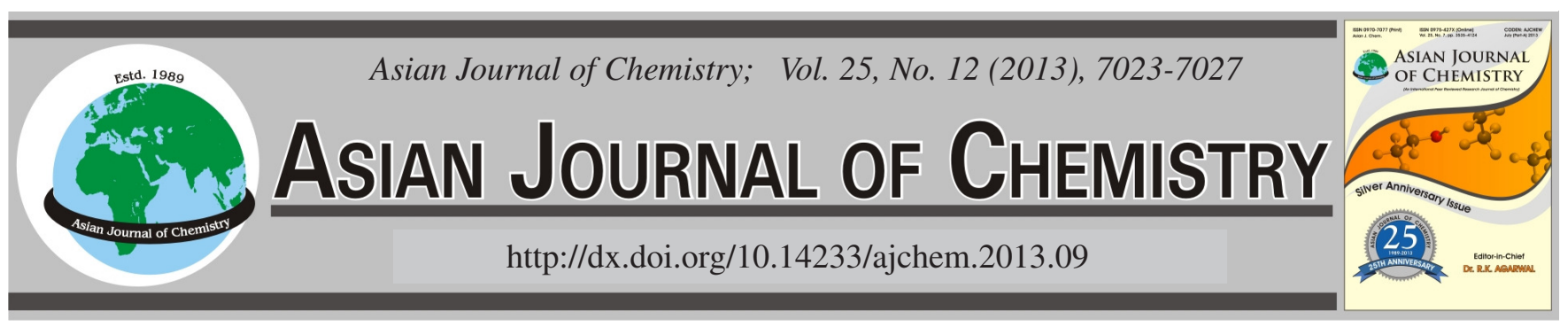

\title{
Effect of Alkyl Alcohol on Viscosity of Silica-Based Chemical Gels for Decontamination of Radioactive Contaminations $\dagger$
}

Suk Bon Yoon, Chang-Ki Kim, Chong-Hun Jung, Byung-Seon Choi , Wang-Kyu Choi, Kune-Woo Lee and Jei-Kwon Moon

Decontamination and Decommissioning Research Division, Korea Atomic Energy Research Institute, 989-111 Daedeok-daero, Yuseong-gu, Daejeon 305-353, Republic of Korea

*Corresponding author: Fax: +82 42 8684797; E-mail: bschoi@kaeri.re.kr

AJC-13631

\begin{abstract}
Silica-based chemical gel for the decontamination of nuclear facilities was prepared using fumed silica as a viscosifier, a $0.5 \mathrm{M}$ Ce (IV) solution dissolved in concentrated nitric acid as a chemical decontamination agent and tripropylene glycol butyl ether (TPGBE) as a coviscosifier. A new effective strategy for the preparation of the chemical gel was investigated by introducing the alkyl alcohols as organic solvents to effectively dissolve the coviscosifier. Using the mixture solution of the coviscosifier and alkyl alcohol was more effective in the control of viscosity than that of only coviscosifier in the gel. Here, alkyl alcohols play a key role as an effective dissolution solvent for the covicosifier in the preparation of the chemical gel, resulting in a reduction of the amount of covicosifier and gel time compared with that of the chemical gel prepared without alkyl alcohols. It was considered that the alkyl alcohols contributed to the effective dissolution of the coviscosifier as well as homogeneous mixing in the formation of the gel, while the coviscosifier in an aqueous media of the chemical decontaminant solution showed a lower solubility. The decontamination efficiency of the chemical gels prepared in this work using a multi-channel analyzer showed a high decontamination efficiency of over $c a .94 \%$ and $c a .92 \%$ for Co-60 and Cs-137 contaminated on the surface of stainless steel 304 , respectively.
\end{abstract}

Key Words: Chemical gel, Fumed silica, Viscosifier, Coviscosifier, Alkyl Alcohol, Decontamination.

\section{INTRODUCTION}

The decontamination is necessary to reduce the radiation field during normal operations and the decommissioning of complex equipment of nuclear facilities. Chemical decontamination technology in particular is a highly effective method to remove radioactive contamination through chemical dissolution or a redox reaction. However, this method has a serious drawback owing to the generation of large amounts of radioactive liquid waste ${ }^{1,2}$.

To avoid the disadvantages of chemical decontamination techniques while retaining their high decontamination efficiency, a few papers have recently reported the preparation of chemical gel decontaminants to reduce the amount of radioactive liquid waste and enhance the decontamination efficiency by increasing the contact time between the gels and the radioactive contaminants ${ }^{3-6}$. In particular, the control of the viscosity depends highly on the amount of coviscosifier used among the components of the gels, which consist of a viscosifier, a coviscosifier and a chemical decontaminant. Gels can be easily prepared through various formulations of viscosifiers, chemical reagents and coviscosifiers. To decontaminate the contaminants of nuclear facilities using the gel, it has to meet such requirements as a thixotropic property, fast drying, easy peel off and non-toxicity. In particular, the thixotropic property is a very important factor in the characteristics of the gels because the gel requires adhesion at a vertical or complex surface and a long contact time between the gel and the contaminant. The gel requirements are summarized in Table- 1 .

\begin{tabular}{ll}
\multicolumn{2}{c}{ TABLE-1 } \\
\multicolumn{2}{c}{ REQUIREMENTS OF THE CHEMICAL GELS } \\
\hline Items & Requirements \\
\hline Thixotropy & $\eta<0.5 \mathrm{~Pa} \cdot \mathrm{s}$ under stress; $4<\eta<8.0 \mathrm{~Pa} \cdot \mathrm{s}$ at rest \\
Suitability & Corrosion resistance, Solubility \\
Drying & $<24 \mathrm{~h}$ \\
Detachability & One-piece or crack \\
Toxicity & Non-toxic \\
\hline
\end{tabular}

In this work, a new and effective method for gel preparation was investigated by introducing various organic solvents such as alkyl alcohols. The mixture solution of coviscosifier and 
alkyl alcohols was more effective in the control of the viscosity compared with that of the coviscosifier only in the gels. The viscosity properties of the gels for the alkyl-chain length in the alkyl alcohols were examined. Furthermore, the decontamination efficiency of the gels was measured using a multichannel analyzer and showed a high decontamination efficiency for Co-60 and Cs-137 contaminated on the surface of stainless steel 304.

\section{EXPERIMENTAL}

Preparation of the chemical gels: Silica-based chemical gel for the decontamination was prepared using fumed silica as a viscosifier, a $0.5 \mathrm{M} \mathrm{Ce}$ (IV) solution dissolved in concentrated nitric acid as a chemical decontamination agent and tripropylene glycol butyl ether as a coviscosifier. In a typical procedure, $2 \mathrm{~g}$ of the fumed silica was added into the reaction bottle containing the decontaminant solution ( $c a .31 .2 \mathrm{~g}$ ) under vigorous stirring. The tripropylene glycol butyl ether of varying amounts was then slowly added after stirring for $0.5 \mathrm{~h}$ and stirred for $3 \mathrm{~h}$ more at ambient condition. To investigate the effect of alkyl alcohol with a different alkyl-chain length such as methanol, ethanol, butanol and hexanol in the formation of the gels, the viscosity of the gels prepared using the mixture solution consisting of tripropylene glycol butyl ether and the alkyl alcohol was compared with that prepared without alkyl alcohol under various experimental conditions such as the amount of tripropylene glycol butyl ether and alkyl alcohol, the stirring time and the stirring speed.

Measurement of the viscosity: The viscosity properties of the gels were measured under conditions of a high shear rate of 500/s for $1 \mathrm{~min}$ (60 points) and a low shear rate of 5/s for $2 \mathrm{~min}$ (120 points), respectively, using a rheometer (Brookfield Eng. and Lab. Inc., RVDV-IIIU) and Rheocalc 32 software.

Investigation of the decontamination efficiency: The contaminated specimens used to investigate the decontamination efficiency of the gels were prepared through the drying of a Co-60 and Cs-137 radionuclide solution on the surface of a SUS 304 plate $(3.5 \mathrm{~cm} \times 3.5 \mathrm{~cm})$ overnight at ambient temperature and calcination for $24 \mathrm{~h}$ at $573 \mathrm{~K}$ using a muffle furnace. The contaminated SUS 304 plates were covered with a thickness of $c a .1 .0 \mathrm{~mm}$ using the gels prepared and dried overnight at ambient temperature. Finally, the dried gels were removed from the contaminated SUS 304 plate. The radioactivity before and after the decontamination was measured using a multi-channel analyzer (Canberra, 2025).

\section{RESULTS AND DISCUSSION}

The fumed silica as a viscosifier exhibited network structures consisting of primary particles with a size of $c a$. 30-50 $\mathrm{nm}$ in diameter, as shown in SEM images (Fig. 1a). In case of using the tripropylene glycol butyl ether only, the viscosity of the gels showed a steep increase over $0.16 \mathrm{~mL}$ of the tripropylene glycol butyl ether (TPGBE) (Fig. 1b). These results imply that the viscosity of the gels is hard to control depending on the amount of coviscosifier used. On the other hand, the viscosity of the gels prepared using the mixture solution of the tripropylene glycol butyl ether/ethyl alcohol was easily controlled (Fig. 1c), showing a gradual slope compared with that of the tripropylene glycol butyl ether only. These results demonstrate that using the mixture solution of a coviscosifier/alcohol is more effective than that of the coviscosifier only because it makes the viscosity easy to control and reduce amount of the coviscosifier.
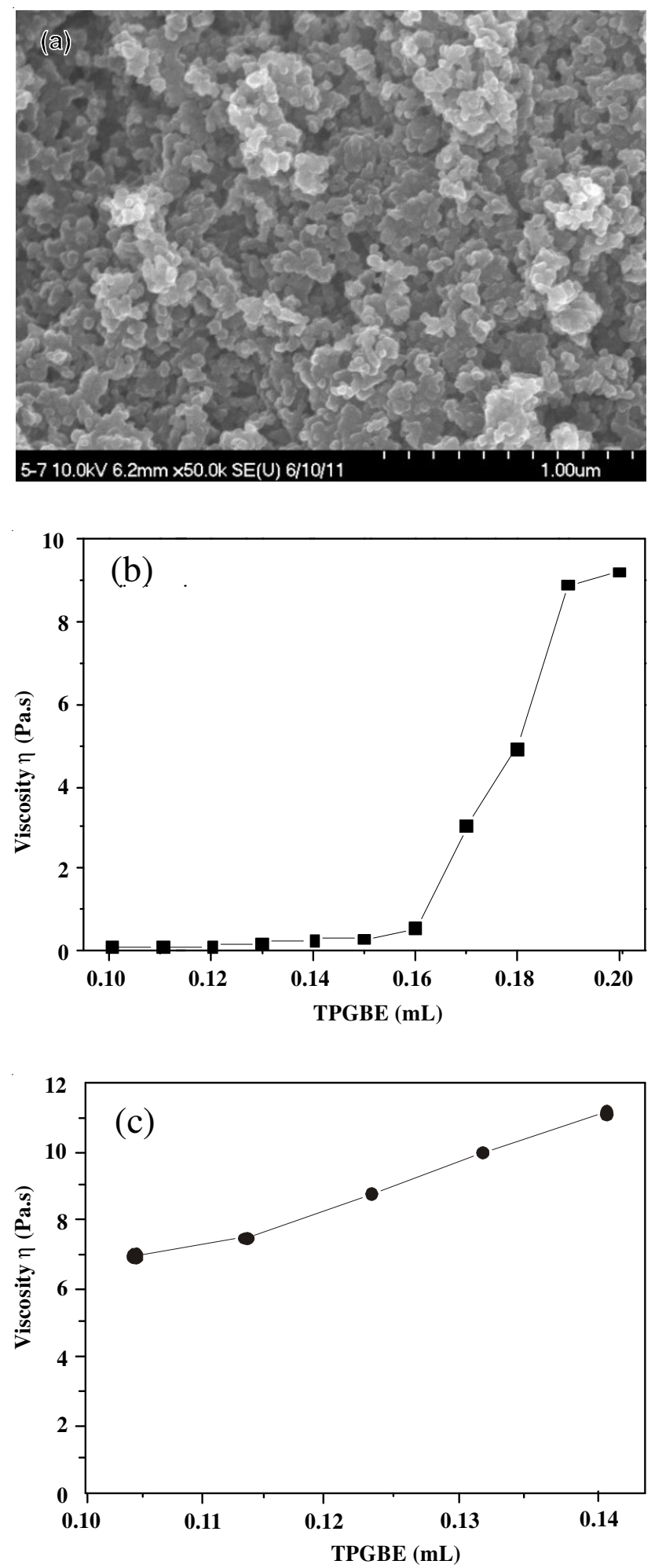

Fig. 1. (a) SEM image of the fumed silica and viscosity properties for the gels prepared by varying the amount of (b) tripropylene glycol butyl ether only and (c) a mixture solution of the tripropylene glycol butyl ether (0.104-0.142 mL)/ethyl alcohol $(0.5 \mathrm{~mL})$ 
From these results (Fig. 1b-c), the ethyl alcohol as an organic solvent played a key role as an effective dissolution solvent for the covicosifier, resulting in a reduction in the amount of covicosifier compared with that of the gel prepared without the ethyl alcohol. It was considered that the ethyl alcohol contributed to the effective dissolution of the coviscosifier as well as the homogeneous mixing in the formation of the gel, while the coviscosifier in aqueous media shows a lower solubility ${ }^{7}$. The influence of the viscosity properties for the stirring time (1-12 h) and speed (250-1000 rpm) was investigated. The resultant gels were nearly independent on the viscosity properties (data not shown). This implies that the preparation of the gels can be completed within $1 \mathrm{~h}$ regardless of the stirring speed.

The effect of alkyl alcohol with a different alkyl-chain length was also investigated. As shown in Fig. 2a, the longer alkyl-chain length showed a higher viscosity property and steeper slope. The viscosity of the gels indicated the highest values when using the amount of methyl alcohol $(1.6 \mathrm{~mL})$, ethyl alcohol $(1.2 \mathrm{~mL})$, butyl alcohol $(0.9 \mathrm{~mL})$ and hexyl alcohol $(0.3 \mathrm{~mL})$, respectively. Also, the viscosity properties for the amount of tripropylene glycol butyl ether used were studied (Fig. 2b). The viscosity of the gels prepared using the methyl alcohol and ethyl alcohol increased with an increase in the amount of tripropylene glycol butyl ether used, showing
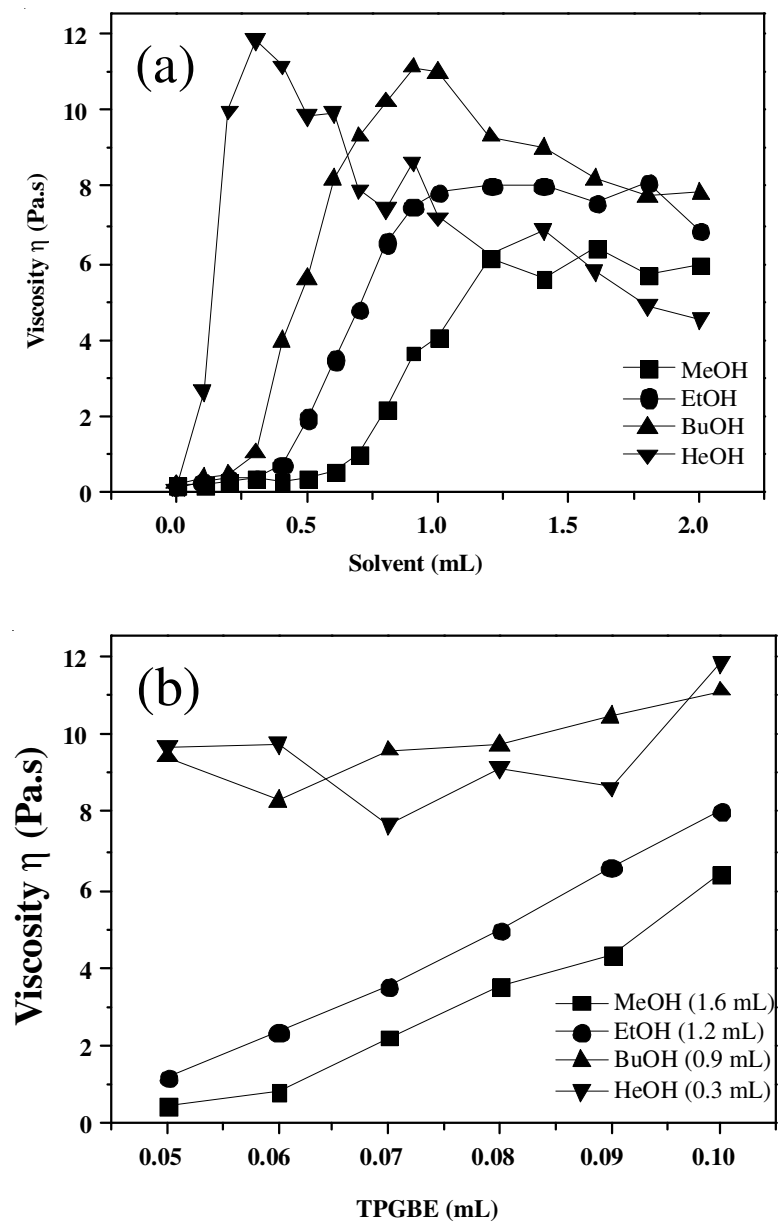

Fig. 2. Viscosity properties of the gels prepared using various alkyl alcohols as organic solvents and the tripropylene glycol butyl ether as a coviscosifier. Control of the amount of (a) alkyl alcohols and (b) tripropylene glycol butyl ether a gentle slope. However, the use of butyl alcohol and hexyl alcohol with a longer alky-chain length showed a similar behaviour of viscosity. This implies that the alkyl-chain of the butyl alcohol and hexyl alcohol provides an additional contribution as a coviscosifier together with the tripropylene glycol butyl ether.

To decontaminate the contaminated surfaces using the gels, the gels must possess a thixotropic property, which is one of the main characteristics in the gels and must be effectively sprayed onto the contaminated surface. The gels with viscosity properties in the range of $c a$. 4.0-8.0 $\mathrm{Pa} \cdot \mathrm{s}$ are selected as the recommended range from the standpoint of good thixotropic behaviour and effective spraying, as mentioned in Table-1. As a result, it can be suggested that the methyl alcohol and ethyl alcohol are more effective than the butyl alcohol and hexyl alcohol for the dissolution solvents of the coviscosifier. Fig. 3 shows rheograms for the gels prepared by varying the amount of tripropylene glycol butyl ether for the alkyl alcohol indicating the highest viscosity properties. Although all gels showed proper viscosity properties below $0.5 \mathrm{~Pa} \cdot \mathrm{s}$ to effectively spray onto the contaminated surface at a high shear rate of 500/s, those of the gels prepared using the butyl alcohol and hexyl alcohol were over $8 \mathrm{~Pa} \cdot \mathrm{s}$ at a low shear rate of $5 / \mathrm{s}$, which does not satisfy the requirements of the chemical gels. A low viscosity of less than $0.5 \mathrm{~Pa} \cdot \mathrm{s}$ at a high shear rate of 500/s results in effective spraying onto the surface, while a high viscosity shows proper surface adhesion at a low shear rate of $5 / \mathrm{s}^{5}$.

The equilibrium viscosity at a low shear rate below $5 / \mathrm{s}$ varies depending on the gel formulation. It was reported that the equilibrium viscosity has an effect on the amount of gelator, chelating agent concentration, type and concentration of the surfactant and the salinity and electrolyte properties of the medium $^{8}$. From the results of the viscosity properties and rheograms, as shown in Fig. 2 and Fig. 3, the gels prepared using methyl alcohol and ethyl alcohol together with tripropylene glycol butyl ether show that they have excellent viscosity properties for a thixotropic behaviour and effective spraying compared with using butyl alcohol and hexyl alcohol, indicating higher viscosity properties over $8.0 \mathrm{~Pa} \cdot \mathrm{s}$.

Fig. 4 shows the results of SEM image, EDX mapping and a spectrum for the cross section of the dried gel prepared using the mixture solution of the tripropylene glycol butyl ether $(0.09 \mathrm{~mL}) /$ ethyl alcohol $(1.2 \mathrm{~mL})$. The thickness of the dried gel was measured as ca $0.67 \mathrm{~mm}$ from the SEM image (Fig. 4a). The EDX mapping revealed a homogeneous element distribution between the viscosifier (M-5) and chemical decontamination agent, ammonium cerium (IV) nitrate $\left[\left(\mathrm{NH}_{4}\right)_{2} \mathrm{Ce}\left(\mathrm{NO}_{3}\right)_{6}\right]$, in the dried gel (Fig. 4b). Peaks corresponding to silicon and oxygen for the M-5 silica and cerium and nitrogen for the $\left(\mathrm{NH}_{4}\right)_{2} \mathrm{Ce}\left(\mathrm{NO}_{3}\right)_{6}$ present in the dried gel can be identified from the results of the EDX spectrum (Fig. 4c).

The decontamination for the SUS 304 plate $(3.5 \mathrm{~cm} \times 3.5$ $\mathrm{cm})$ contaminated with Co-60 and Cs-137 radionuclides was investigated. The gel prepared using the mixture solution of tripropylene glycol butyl ether $(0.09 \mathrm{~mL})$ /ethyl alcohol $(1.2$ $\mathrm{mL}$ ) was used for the decontamination owing to a proper thixotropic behaviour and effective spraying. The decontamination 

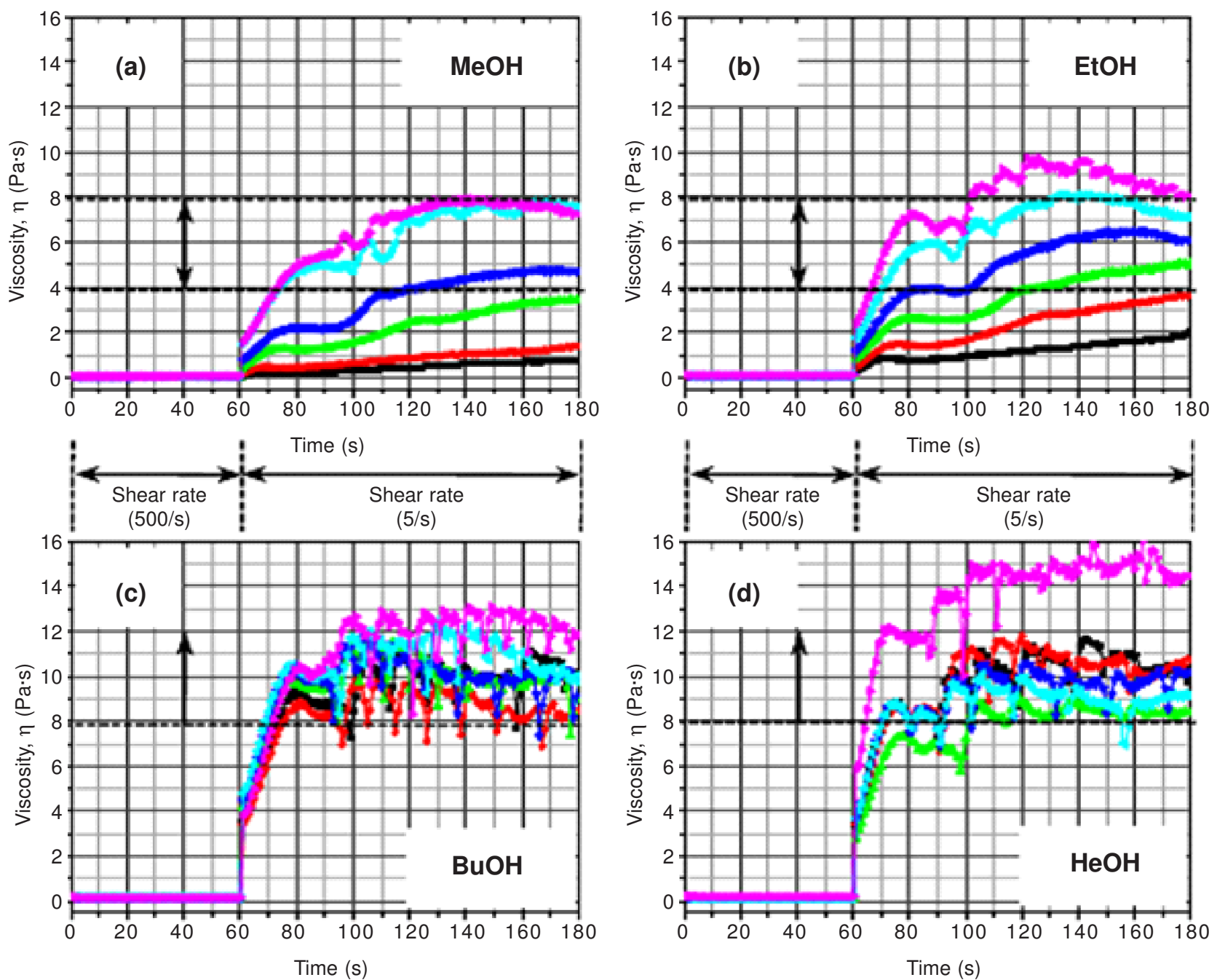

Fig. 3. Representative rheograms for the gels prepared by the control of the amount of tripropylene glycol butyl ether and alkyl alcohols of (a) methyl alcohol $(1.6 \mathrm{~mL})$, (b) ethyl alcohol $(1.2 \mathrm{~mL})$, (c) buty alcohol $(0.9 \mathrm{~mL})$ and $(\mathrm{d})$ hexyl alcohol $(0.3 \mathrm{~mL})$. The symbols represent the amount of tripropylene glycol butyl ether used [0.05 $\mathrm{mL}(\boldsymbol{\square}), 0.06 \mathrm{~mL}(\mathbf{\bullet}), 0.07 \mathrm{~mL}(\mathbf{\Lambda}), 0.08 \mathrm{~mL}(\boldsymbol{\nabla}), 0.09 \mathrm{~mL}(\triangleleft)$ and $0.10 \mathrm{~mL}(\mathbf{)})]$
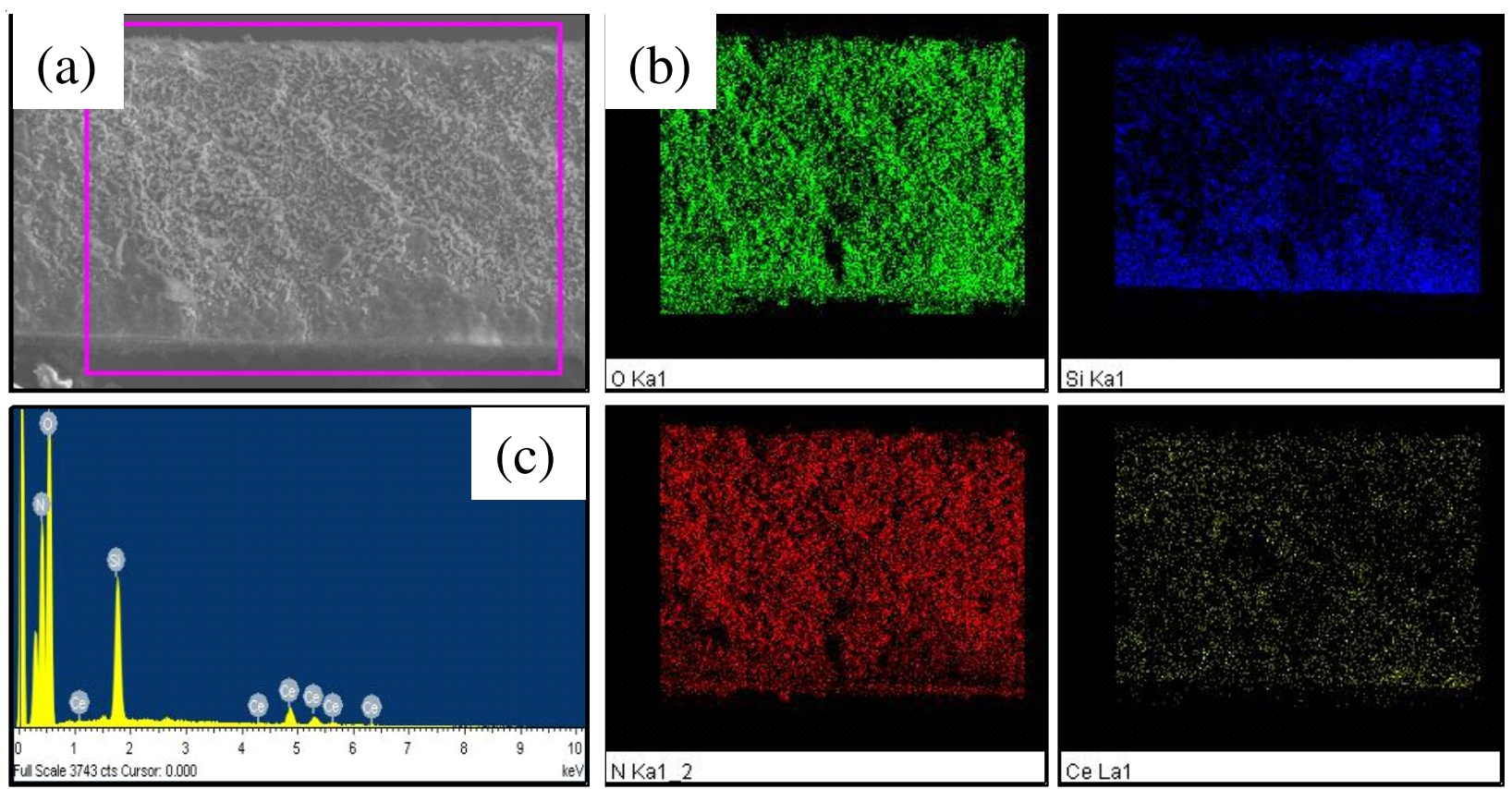

Fig. 4. (a) SEM image, (b) EDX mapping and (c) EDX spectrum, for the cross section of the dried gel prepared using the mixture solution of the tripropylene glycol butyl ether $(0.09 \mathrm{~mL}) /$ ethyl alcohol $(1.2 \mathrm{~mL})$ 
efficiency of the gels using a multi-channel analyzer (MCA) showed a high decontamination efficiency of over ca. $94 \%$ and ca. $92 \%$ for the Co-60 and Cs-137 radionuclides, respectively. The results of the multi-channel analyzer measurement for the gels are summarized in Table-2.

TABLE-2

RESULTS OF THE MCA MEASUREMENT FOR THE GELS PREPARED USING THE TPGBE/EtOH MIXTURE SOLUTION

\begin{tabular}{ccccccc}
\hline Sample & \multicolumn{3}{c}{ Co-60 } & \multicolumn{3}{c}{ Cs-137 } \\
\cline { 2 - 7 } No. & Before & After & DF $(\%)$ & Before & After & DF $(\%)$ \\
\hline 1 & 338 & 8.5 & 97.8 & 650 & N/D & 100 \\
2 & 214 & 12.9 & 94.0 & 447 & N/D & 100 \\
3 & 185 & 8.3 & 95.5 & 632 & 51.5 & 91.9 \\
4 & 319 & 18.0 & 94.4 & 526 & N/D & 100 \\
\hline
\end{tabular}

\section{Conclusion}

The use of an organic solvent together with a coviscosifier is very effective owing to the dissolution of the coviscosifier as well as the homogeneous mixing in the formation of the gel, resulting in a reduction in the amount of covicosifier compared with that of a gel prepared without an organic solvent. The viscosity property of the gels was easily controlled more than the use of the coviscosifier only. The gels prepared using the mixture solution of the coviscosifier and organic solvents with a short alkyl-chain length, such as methyl alcohol and ethyl alcohol in particular, exhibited proper viscosity properties for a good thixotropic behaviour and effective spraying compared with the use of a long alkyl-chain length. The decontamination efficiency of the gels showed a high decontamination efficiency for the surfaces contaminated with Co60 and Cs-137 radionuclides on the surface of stainless steel 304 , respectively. Further research for the preparation of the gels using various organic solvents is currently under progress.

\section{ACKNOWLEDGEMENTS}

This work was performed as part of the Nuclear Research and Development Program of the Ministry of Education, Science and Technology.

\section{REFERENCES}

1. A.L. Taboas, A.A. Moghissi and T.S. La Guardia, ASME, pp. 4-22 (2004).

2. IAEA, State of the Art Technology for Decontamination and Dismantling of Nuclear Facilities, IAEA in Austria (1999).

3. L. Nunez and M.D. Kaminski, Foam and Gel Methods for the Decontamination of Metallic Surfaces, US Patent, US 2006/0217584 A1 (2006).

4. S. Faure, P. Fuentes and Y. Lallot, Vacuumable Gel for Decontaminating Surfaces and Use Thereof. US Patent, US 2008/0228022 A1 (2008).

5. C.H. Jung, J.K. Moon, H.J. Won, K.W. Lee and C.K. Kim, Chemical Gel for Surface Decontamination. Transactions of the Korean Nuclear Society Autumn Meeting, Jeju, Korea, October, 21-22 (2010).

6. Feltcorn, Technology Reference Guide for Radiologically Contaminated Surfaces. U.S. Envirnmental Protection Agency (2006).

7. J.N. Coupland, D. Brathwaite, P. Fairley and D.J. McClements, J. Colloid Interf. Sci., 190, 71 (1997).

8. L. Nunez and M.D. Kaminski, Foam and Gel Methods for the Decontamination of Metallic Surfaces, US Patent, US Patent No. 7166758, (2007). 